\title{
\begin{tabular}{l|l} 
Mitraries & DSpace@MIT
\end{tabular}
}

\author{
MIT Open Access Articles
}

Role of surface oxygen-to-metal ratio
on the wettability of rare-earth oxides

The MIT Faculty has made this article openly available. Please share how this access benefits you. Your story matters.

Citation: Khan, Sami, Gisele Azimi, Bilge Yildiz, and Kripa K. Varanasi. “Role of Surface Oxygento-Metal Ratio on the Wettability of Rare-Earth Oxides." Appl. Phys. Lett. 106, no. 6 (February 9 , 2015): 061601. (C) 2015 AIP Publishing LLC

As Published: http://dx.doi.org/10.1063/1.4907756

Publisher: American Institute of Physics (AIP)

Persistent URL: http://hdl.handle.net/1721.1/97737

Version: Final published version: final published article, as it appeared in a journal, conference proceedings, or other formally published context

Terms of Use: Article is made available in accordance with the publisher's policy and may be subject to US copyright law. Please refer to the publisher's site for terms of use. 


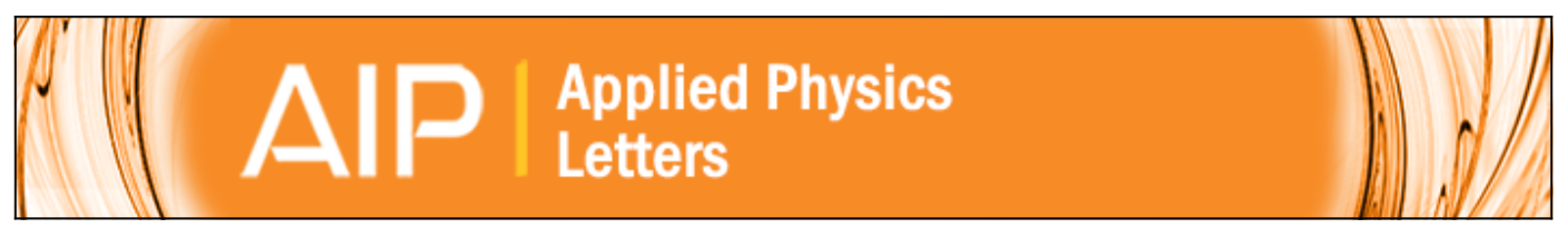

\section{Role of surface oxygen-to-metal ratio on the wettability of rare-earth oxides}

Sami Khan, Gisele Azimi, Bilge Yildiz, and Kripa K. Varanasi

Citation: Applied Physics Letters 106, 061601 (2015); doi: 10.1063/1.4907756

View online: http://dx.doi.org/10.1063/1.4907756

View Table of Contents: http://scitation.aip.org/content/aip/journal/apl/106/6?ver=pdfcov

Published by the AIP Publishing

\section{Articles you may be interested in}

Effect of hydrocarbon adsorption on the wettability of rare earth oxide ceramics

Appl. Phys. Lett. 105, 011601 (2014); 10.1063/1.4886410

The effect of PECVD plasma decomposition on the wettability and dielectric constant changes in silicon modified DLC films for potential MEMS and low stiction applications

AlP Advances 2, 032128 (2012); 10.1063/1.4742852

Facile creation of bio-inspired superhydrophobic Ce-based metallic glass surfaces

Appl. Phys. Lett. 99, 261905 (2011); 10.1063/1.3672036

Wettability and "petal effect" of GaAs native oxides

J. Appl. Phys. 110, 034319 (2011); 10.1063/1.3619797

Ternary rare-earth metal oxide high- $\mathrm{k}$ layers on silicon oxide

Appl. Phys. Lett. 86, 132903 (2005); 10.1063/1.1886249

\section{HDDEN}

\section{Instruments for Advanced Science}

Contact Hiden Analytical for further details: w www.HidenAnalytical.com E info@hiden.co.uk CLICK TO VIEW our product catalogue

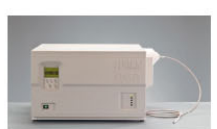

Gas Analysis

dynamic measurement of reaction gas streams catalysis and thermal analysis

molecular beam studies

, dosolved specis probss

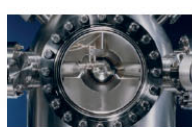

Surface Science

, UHVTPD

SIMS end point detection in ion beam etch

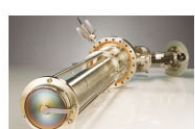

Plasma Diagnostics

- plasma source characterization etch and deposition process reaction kinetic studies , analysis of neutral and radical species

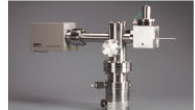

Vacuum Analysis partial pressure measurement and control of process gases reactive sputter process contro , vacuum diagnostics Tacum coating process monitoring 


\title{
Role of surface oxygen-to-metal ratio on the wettability of rare-earth oxides
}

\author{
Sami Khan, ${ }^{1}$ Gisele Azimi, ${ }^{2}$ Bilge Yildiz, ${ }^{3}$ and Kripa K. Varanasi ${ }^{1, a)}$ \\ ${ }^{1}$ Department of Mechanical Engineering, Massachusetts Institute of Technology, Cambridge, \\ Massachusetts 02139, USA \\ ${ }^{2}$ Department of Chemical Engineering and Applied Chemistry and Materials Science and Engineering, \\ University of Toronto, Toronto, Ontario MSS 3E5, Canada \\ ${ }^{3}$ Department of Nuclear Science and Engineering, Massachusetts Institute of Technology, Cambridge, \\ Massachusetts 02139, USA
}

(Received 9 December 2014; accepted 25 January 2015; published online 9 February 2015)

\begin{abstract}
Hydrophobic surfaces that are robust can have widespread applications in drop-wise condensation, anti-corrosion, and anti-icing. Recently, it was shown that the class of ceramics comprising the lanthanide series rare-earth oxides (REOs) is intrinsically hydrophobic. The unique electronic structure of the rare-earth metal atom inhibits hydrogen bonding with interfacial water molecules resulting in a hydrophobic hydration structure where the surface oxygen atoms are the only hydrogen bonding sites. Hence, the presence of excess surface oxygen can lead to increased hydrogen bonding and thereby reduce hydrophobicity of REOs. Herein, we demonstrate how surface stoichiometry and surface relaxations can impact wetting properties of REOs. Using X-ray Photoelectron Spectroscopy and wetting measurements, we show that freshly sputtered ceria is hydrophilic due to excess surface oxygen (shown to have an $\mathrm{O} / \mathrm{Ce}$ ratio of $\sim 3$ and a water contact angle of $\sim 15^{\circ}$ ), which when relaxed in a clean, ultra-high vacuum environment isolated from airborne contaminants reaches close to stoichiometric O/Ce ratio $(\sim 2.2)$ and becomes hydrophobic (contact angle of $\sim 104^{\circ}$ ). Further, we show that airborne hydrocarbon contaminants do not exclusively impact the wetting properties of REOs, and that relaxed REOs are intrinsically hydrophobic. This study provides insight into the role of surface relaxation on the wettability of REOs. (C) 2015 AIP Publishing LLC.
\end{abstract}

[http://dx.doi.org/10.1063/1.4907756]

Superhydrophobic surfaces, which require a combination of surface chemistry and surface topography, have attracted significant attention over the past decade because of their broad technological potential. ${ }^{1-5}$ There has been considerable focus on understanding their wettability 6,7 and developing them for a wide range of applications including droplet impact resistance, ${ }^{8-13}$ anti-icing, ${ }^{14-19}$ dropwise condensation, ${ }^{20-24}$ electro-wetting, ${ }^{25,26}$ drag reduction, ${ }^{27-32}$ evaporation, ${ }^{33,34}$ and anti-corrosion. ${ }^{35-38}$ An important challenge for broad applicability of these hydrophobic materials is their limited robustness. ${ }^{3,4,39}$ Most approaches for fabricating superhydrophobic surfaces involve texturing a polymeric hydrophobic material or coating a textured hydrophilic material with a polymeric hydrophobic modifier such as organosilanes or thiols. ${ }^{40}$ Existing durable materials such as metals and ceramics are generally hydrophilic and require polymeric hydrophobic modifiers to render them hydrophobic. ${ }^{41}$ However, polymeric modifiers deteriorate in harsh environments making it difficult to realize robust hydrophobic surfaces. Hence, there is a clear need for robust materials such as ceramics that are intrinsically hydrophobic.

Recent studies have shown that the class of ceramics comprising the lanthanide series rare-earth oxides (REOs) is intrinsically hydrophobic. ${ }^{42-47}$ REOs are capable of sustaining their intrinsic hydrophobicity after exposure to extreme processing conditions such as high temperature, abrasive wear, and steam. ${ }^{42}$ The underlying phenomenon of hydrophobicity

\footnotetext{
${ }^{\text {a) }}$ Author to whom correspondence should be addressed. Electronic mail: varanasi@mit.edu
}

in REOs arises from the unique electronic structure of the rare-earth metal atoms where the unfilled $4 f$ orbitals are shielded from interactions with the environment by a full octet of electrons in the $5 s^{2} p^{6}$ outer shell. ${ }^{42}$ This shielding effect minimizes the hydrogen bonding with interfacial water molecules and renders REOs hydrophobic ${ }^{42}$ as shown in Figure 1. In contrast, in the case of a common ceramic like alumina, the lack of such a shielding effect allows the metal
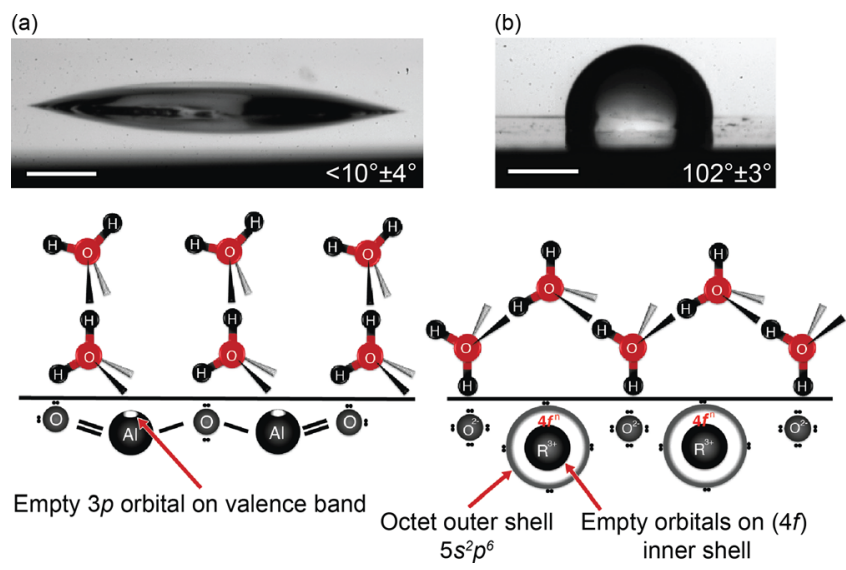

FIG. 1. Schematic of the orientation of water molecules and the associated wetting properties of a surface. (a) Hydrophilicity and schematic of the orientation of water molecules next to an alumina surface (using different scales for the surface and water molecules). (b) Hydrophobicity and schematic of the orientation of water molecules next to neodymia (an REO) (surface and water molecules not to scale). Scale bars, $1 \mathrm{~mm}$. Reprinted with permission from Azimi et al., Nat. Mater. 12, 315 (2013). Copyright 2013 Macmillan Publishers Limited. 
atom to hydrogen bond with interfacial water molecules. ${ }^{48,49}$ In this case, water molecules are able to maintain their hydrogenbonding network on alumina, resulting in hydrophilicity.

The surface chemistry of REOs plays a significant role in determining the wettability. If the REO surface were to be modified in that the surface lattice oxygen sites were increased relative to the rare-earth metal sites, its hydrophobicity will be affected. In other words, a net increase in the surface oxygen-to-metal ratio beyond the typical stoichiometric value is detrimental because an increase in surface lattice oxygen provides more avenues for hydrogen-bonding with interfacial water which further breaks down the hydrophobic hydration structure seen in Figure 1, thereby increasing wettability. In the case of ceria $\left(\mathrm{CeO}_{2}\right)$ studied in this paper, an $\mathrm{O} / \mathrm{Ce}$ ratio higher than 2.0 can negatively impact wettability, as it provides more sites for hydrogen bonding with water.

Furthermore, recent literature has addressed the contribution of surface hydrocarbons arising from natural airborne contamination towards wettability of REOs. ${ }^{50}$ Researchers have shown that the contact angle of water measured on REOs increases with time when left in ambient air as it progressively adsorbs atmospheric hydrocarbon contaminants. ${ }^{50}$ While it is important to avoid ruling out the effect of adsorbed surface hydrocarbon contaminants on the wettability of REOs, it is also important to study how changes in surface chemistry and stoichiometry of a pristine REO surface potentially impact wettability.

In this paper, we show how surface relaxation plays an important role in determining the wettability of REOs. We first show that hydrophobicity of REOs is not exclusively dominated by surface hydrocarbon contamination. We then show that surface relaxation in a low carbon, ultra-high vacuum (UHV) environment causes a reduction in the surface oxygen-to-metal ratio in REOs owing to changes in both oxygen and rare-earth metal chemistry. Finally, we show that a decrease in the surface oxygen-to-metal ratio in REOs is accompanied by a corresponding increase in water contact angle (WCA), and thereby we demonstrate the crucial importance of surface relaxations in ascertaining the hydrophobicity of REOs. Ceria was chosen as a model REO in this study given the extensive past literature on surface chemistry and relaxation of ceria. Henceforth, we will define surface relaxation as the change in surface stoichiometry.

In order to better understand the contribution of surface hydrocarbon contamination towards the hydrophobicity of REOs, we first compared and contrasted ceria and alumina under identical conditions (which, as described earlier, are intrinsically hydrophobic and hydrophilic, respectively). We sputter-deposited approximately $300 \mathrm{~nm}$ thick layers of ceria and alumina on distinct silicon wafers (AJAsputterer ATC series) and transported both samples in the same vacuum desiccator for X-Ray Photoelectron Spectroscopy (XPS) analysis in an UHV chamber (PHI Versaprobe II XPS). Immediately after XPS measurements, WCAs were measured using a goniometer (Rame-Hart M500 series). As shown in Table I, both surfaces were found to have similar surface carbon content $(\sim 15 \%)$ measured by XPS, yet only ceria was hydrophobic $\left(\mathrm{WCA}>90^{\circ}\right.$ ), while alumina was hydrophilic $\left(\mathrm{WCA} \ll 90^{\circ}\right.$ ). These results clearly indicate
TABLE I. Effect of surface carbon content: Comparison between ceria and alumina.

\begin{tabular}{lcc}
\hline \hline Surface & Surface atomic carbon content $(\%)$ & Contact angle (deg) \\
\hline Ceria & $15.1 \pm 2.1$ & $104 \pm 2$ \\
Alumina & $15.8 \pm 2.5$ & $45 \pm 3$ \\
\hline \hline
\end{tabular}

that surface hydrocarbon contamination exclusively cannot explain the hydrophobicity of REOs. In fact, the ceria sample was hydrophobic despite having an almost two-fold lower surface carbon content $(\sim 15 \%)$ than previously reported values on a hydrophobic ceria surface exposed to ambient air $(\sim 34 \%)$. $^{50}$

Having demonstrated that surface carbon does not uniquely explain the hydrophobicity of ceria, we then focused on better understanding the surface chemistry of ceria and the phenomenon of surface relaxation. There are many factors that could potentially cause a departure from the pristine stoichiometric state of ceria. Chin et al. ${ }^{51}$ found that freshly sputter-deposited ceria films have an overstoichiometric $\mathrm{O} / \mathrm{Ce}$ ratio on the surface, reaching as high as 3.3. They attributed this anomaly to cerium vacancies or interstitial excess oxygen on the surface arising from the sputtering process. Such surfaces need to undergo long-term relaxation to reach an optimal surface stoichiometry before intrinsic hydrophobicity can be truly ascertained.

If REOs were made to relax in ambient air, atmospheric hydrocarbon contaminants would potentially accumulate on the surface and hence interfere with genuine intrinsic hydrophobicity. Hence, in order to observe surface relaxation in an environment isolated from airborne hydrocarbon contaminants, we used a UHV chamber that was maintained at a vacuum of $1.4 \times 10^{-10}$ to $5.5 \times 10^{-10}$ Torr. We sputter-deposited a $300 \mathrm{~nm}$ thick layer of ceria on a silicon wafer and immediately transferred the sample in a vacuum desiccator to the UHV chamber. In situ XPS analyses were conducted in the same chamber at regular time intervals for $6 \mathrm{~h}$ after which the sample was left to relax in the chamber overnight for $14 \mathrm{~h}$, following which a final XPS spectrum was obtained.

Figures 2(a) and 2(b) compare the high-resolution XPS spectra for cerium (Ce 3d) obtained before and after overnight UHV relaxation, respectively. It can be seen that the shape of the cerium spectrum changed considerably after overnight relaxation for $14 \mathrm{~h}$ in UHV. To investigate this change in detail, the Ce $3 \mathrm{~d}$ spectra were deconvoluted into constituent satellite peaks and identified using the nomenclature proposed by Burroughs et al. ${ }^{52}$ Specifically, the satellite peaks were designated with two different letters for the two $3 \mathrm{~d}$ components of Ce $3 \mathrm{~d} ; v$ for $3 \mathrm{~d}_{5 / 2}$ peaks and $u$ for $3 \mathrm{~d}_{3 / 2}$. All constituent satellite peaks of Ce $3 d$ were identified by this method as shown in Figures 2(a) and 2(b) (with the exception of $u_{o}$ and $v_{o}$ which could not be resolved at their characteristic peak position due to lack of intensity). The peaks were fitted according to the constraint that the intensity ratio between each $v$ peak and its corresponding $u$ peak should equal $1.5 .^{53,54}$

The peaks were then associated with the $\mathrm{Ce}^{4+}$ or the $\mathrm{Ce}^{3+}$ oxidation state. Peaks $v, v^{\prime \prime}, v^{\prime \prime \prime}, u, u^{\prime \prime}$, and $u^{\prime \prime \prime}$ were associated with the $\mathrm{Ce}^{4+}$ state, while $v_{0}, v^{\prime}, u_{0}$, and $u^{\prime}$ were 
(a)

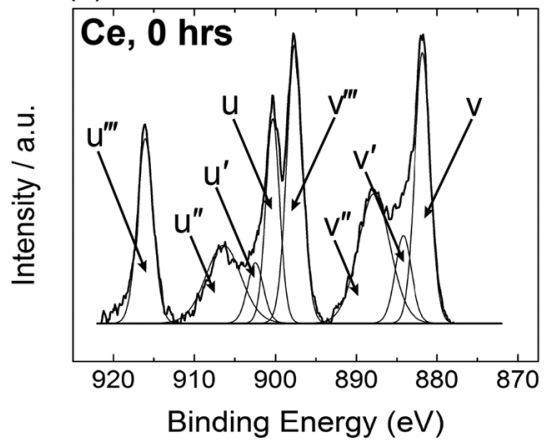

(c)

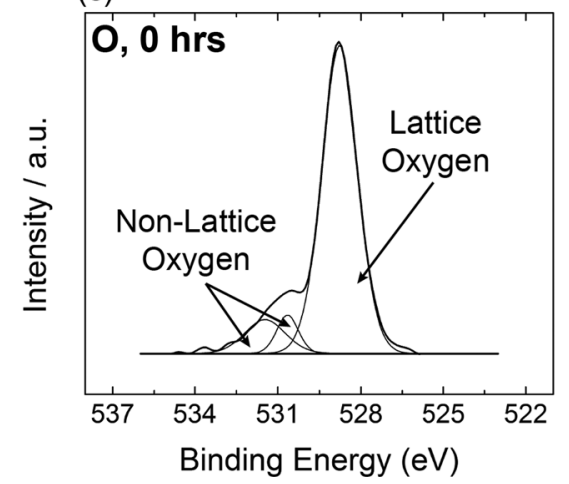

(b)

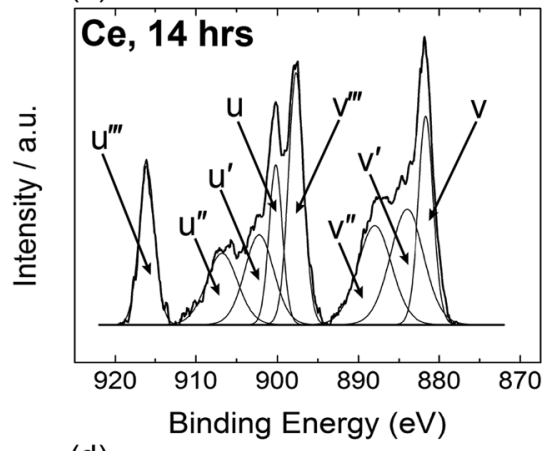

(d)

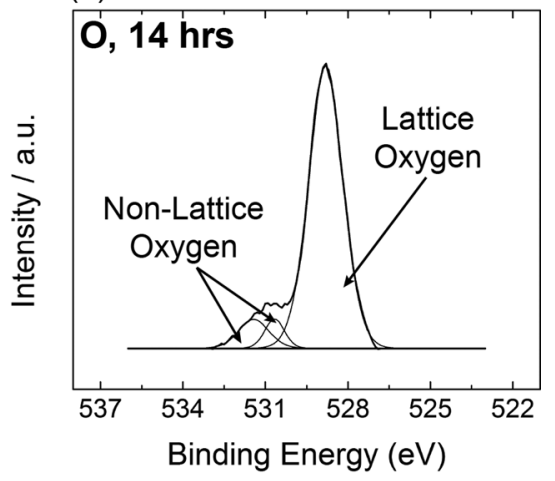

FIG. 2. (a) XPS spectrum of cerium (Ce 3d) with constituent satellite peaks obtained immediately after loading in the UHV chamber. (b) XPS spectrum of cerium (Ce $3 d$ ) with constituent satellite peaks obtained after $14 \mathrm{~h}$ relaxation in UHV. (c) XPS spectrum of oxygen (O 1s) immediately after loading in the UHV chamber. (d) XPS spectrum of oxygen (O 1s) obtained after $14 \mathrm{~h}$ relaxation in $\mathrm{UHV}$. associated with the $\mathrm{Ce}^{3+}$ state. $^{52-56}$ The relative concentrations of $\mathrm{Ce}^{4+}$ and $\mathrm{Ce}^{3+}$ were determined using the following equations: ${ }^{52-56}$

$$
\begin{gathered}
A\left(\mathrm{Ce}^{3+}\right)=A\left(v_{0}\right)+A\left(v^{\prime}\right)+A\left(u_{0}\right)+A\left(u^{\prime}\right), \\
A\left(\mathrm{Ce}^{4+}\right)=A(v)+A\left(v^{\prime \prime}\right)+A\left(v^{\prime \prime \prime}\right)+A(u)+A\left(u^{\prime \prime}\right)+A\left(u^{\prime \prime \prime}\right), \\
\% \mathrm{Ce}^{y+}=\frac{A\left(\mathrm{Ce}^{y+}\right)}{A\left(\mathrm{Ce}^{3+}\right)+A\left(\mathrm{Ce}^{4+}\right)} \times 100 \quad(y=3,4)
\end{gathered}
$$

$A(x)$ above refers to the peak area (and corresponding peak intensity) as determined from the XPS spectra.

Using the above equations, it was determined that the $\mathrm{Ce}^{3+}$ contribution to the overall surface cerium increased from $10.2 \%$ to $27.2 \%$ with overnight relaxation in UHV, while the $\mathrm{Ce}^{4+}$ contribution decreased from $89.8 \%$ to $72.8 \%$. This indicates that the ceria sample underwent a partial reduction in state from $\mathrm{Ce}^{4+}$ to $\mathrm{Ce}^{3+}$ in $\mathrm{UHV}$, which is consistent with observations of Zhang et al. ${ }^{55}$ Specifically, it can be seen in Figures 2(a) and 2(b) that the intensity of both the characteristic $\mathrm{Ce}^{3+}$ peaks $v^{\prime}$ and $u^{\prime}$ increased following overnight relaxation in $\mathrm{UHV}$, while the characteristic $\mathrm{Ce}^{4+}$ peaks $v, v^{\prime \prime}$, $v^{\prime \prime \prime}, u, u^{\prime \prime}$, and $u^{\prime \prime \prime}$ all decreased in intensity. Preisler et al. ${ }^{57}$ have further correlated the change in the oxidation state from $\mathrm{Ce}^{4+}$ to $\mathrm{Ce}^{3+}$ with a net decrease in the $\mathrm{O} / \mathrm{Ce}$ ratio. Therefore, in view of all these observations, we can say, that the change in cerium chemistry, as seen in Figures 2(a) and 2(b), is consistent with the hypothesis that surface relaxation in UHV caused an overall decrease in the surface $\mathrm{O} / \mathrm{Ce}$ ratio.

It can be further seen from Figures 2(c) and 2(d) that the intensity of both the lattice and non-lattice oxygen peaks decreased with overnight relaxation in UHV. It is known in the literature that ceria can release surface lattice oxygen as part of a wider surface reconstruction process. As an example, Solovyov et al. ${ }^{58}$ studied oxygen reconstruction on an unstable [001] ceria surface. Such surfaces release lattice oxygen in order to maintain a net zero dipole moment perpendicular to the surface, which potentially explains the reason for decreasing lattice oxygen sites as observed by the reduction in the lattice oxygen peak intensity after overnight relaxation in UHV. Therefore, the change in the surface oxygen is consistent with the hypothesis that surface relaxation in UHV caused an overall decrease in the surface $\mathrm{O} / \mathrm{Ce}$ ratio.

The non-lattice oxygen peaks at $530.7 \mathrm{eV}$ and $531.4 \mathrm{eV}$ in Figures 2(c) and 2(d) have been previously attributed to adsorbed surface oxygen species such as hydroxyls or carbonates. ${ }^{44,59,60}$ Furthermore, the characteristic O 1s peak associated with adsorbed molecular water at $532.9 \mathrm{eV}$ was not observed. Martínez et al. ${ }^{44}$ on the contrary observed very intense peaks for adsorbed surface oxygen and molecular water relative to the lattice oxygen. Thus, the low concentration of adsorbed surface oxygen species relative to the lattice oxygen as well as the lack of adsorbed molecular water confirms the low degree of surface contamination on our ceria sample. This is attributable to the quick transfer of the sputtered samples to the UHV chamber under vacuum as well as the clean, isolated nature of the UHV environment.

The high-resolution XPS spectra were used to determine the relative surface atomic concentrations of cerium, oxygen, and carbon. Figure 3(a) shows that as the sample relaxed in $\mathrm{UHV}$, the surface $\mathrm{O} / \mathrm{Ce}$ ratio steadily decreased from 3.0 to 2.2 over the $14 \mathrm{~h}$ of the experiment, reaching close to the stoichiometric ratio of ceria. The surface carbon remained steady at $\sim 12 \%$ throughout the experiment as seen in Figure 3(b) which was expected given that the UHV chamber was practically devoid of hydrocarbon contaminants and well sealed from the ambient environment.

How does the decreasing $\mathrm{O} / \mathrm{Ce}$ ratio impact wettability? As explained previously in Figure 1, greater the oxygen sites 
(a)

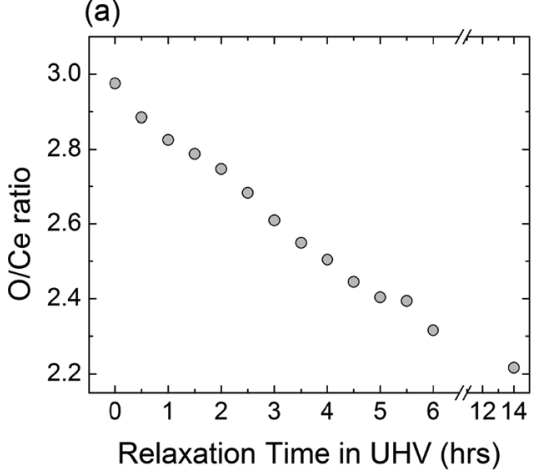

(c)

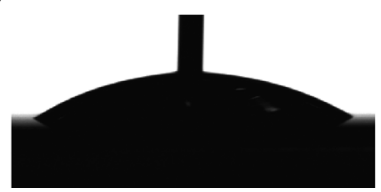

(b)

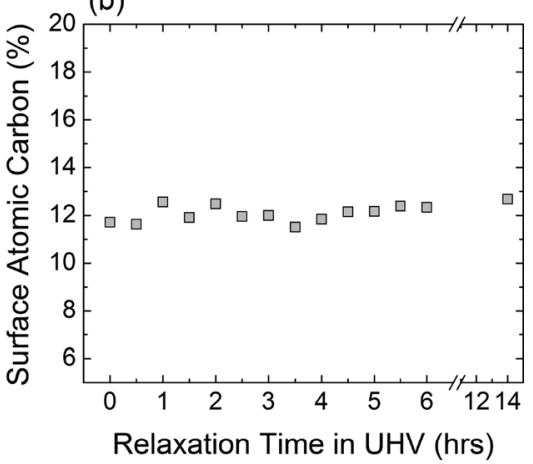

(d)

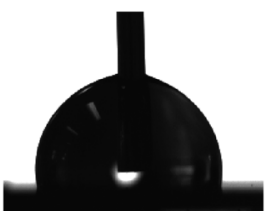

FIG. 3. (a) Effect of UHV relaxation of a sputtered ceria surface on the $\mathrm{O} / \mathrm{Ce}$ ratio. The $\mathrm{O} / \mathrm{Ce}$ ratio decreases from $\sim 3.0$ at the start of the analysis to $\sim 2.2$ after $14 \mathrm{~h}$ in a UHV chamber. (b) Variation of surface atomic carbon concentration with relaxation time in UHV. (c) Advancing water contact angle on a freshly sputtered ceria surface with an $\mathrm{O} / \mathrm{Ce}$ ratio of $\sim 3.0$ measured as $15^{\circ}$ using a goniometer. (d) Advancing water contact angle on a UHV-relaxed ceria surface with an $\mathrm{O} / \mathrm{Ce}$ ratio of $\sim 2.2$ measured as $104^{\circ}$ using a goniometer. on the surface, the greater the tendency to form hydrogen bonds with interfacial water and hence higher the wettability. As seen in Figure 3(a), the O/Ce ratio on a freshly sputterdeposited ceria surface was measured as $\sim 3.0$, which is similar to observations of Chin et al. ${ }^{51}$ Indeed, we observed that water spreads on a freshly sputter-deposited ceria surface as shown in Figure 3(c) given the higher-than-stoichiometric $\mathrm{O} / \mathrm{Ce}$ ratio. However, when this surface was allowed to relax in a UHV environment, the $\mathrm{O} / \mathrm{Ce}$ ratio approaches the stoichiometric ratio and the surface is rendered hydrophobic $\left(\mathrm{WCA}>90^{\circ}\right.$ ) as shown in Figure 3(d). Hence, because of the extensive surface relaxation in the form of decreasing $\mathrm{O} / \mathrm{Ce}$ ratio, the ceria surface transitioned from being initially hydrophilic to hydrophobic. It is important to note that this transition occurred with a negligible change in surface carbon content. This demonstrates that surface relaxation and determining the $\mathrm{O} / \mathrm{Ce}$ ratio are crucial towards ascertaining hydrophobicity of REOs and surface hydrocarbons do not exclusively explain this unique phenomenon. This is further consistent with contact angle measurements confirming hydrophobicity on the relaxed sample with a close-to-stoichiometric $\mathrm{O} / \mathrm{Ce}$ ratio and is overall consistent with our theory on wetting behavior of REOs. Table II summarizes the results from the start and end points of the surface relaxation experiment.

In summary, REOs are intrinsically hydrophobic and we show how excess surface oxygen can negatively impact hydrophobicity. More broadly, we show how surface chemistry (in particular, excess surface oxygen) and surface relaxations can impact wettability of REOs. In fact, contact angle measurements can be used as a tool to determine the extent of surface relaxation of REOs. Further, we show that airborne hydrocarbon contaminants do not exclusively impact

TABLE II. Comparison of water contact angles, O/Ce ratio, and surface carbon content on freshly sputtered and UHV-relaxed ceria samples.

\begin{tabular}{lrrr}
\hline \hline Sample & Advancing WCA (deg) & O/Ce ratio & Surface carbon (\%) \\
\hline As-sputtered & $15 \pm 6$ & $2.98 \pm 0.04$ & $11.7 \pm 0.3$ \\
UHV-relaxed (14h) & $104 \pm 3$ & $2.22 \pm 0.02$ & $12.7 \pm 0.2$ \\
\hline \hline
\end{tabular}

wetting properties of REOs. Hence, it is important to carefully consider these effects before ascertaining the true origins of hydrophobicity of REOs.

We gratefully acknowledge financial support from the NSF Career Award (Project Number: 0952564), the MIT-KFUPM Center for Clean Water and Clean Energy under Project \#MIT-KFUPM-R16, and the Hydro Research Foundation Graduate Award.

${ }^{1}$ P.-G. de Gennes, F. Brochard-Wyart, and D. Quere, Capillarity and Wetting Phenomena: Drops, Bubbles, Pearls, Waves (Springer Science \& Business Media, 2004).

${ }^{2}$ H.-J. Butt, K. Graf, and M. Kappl, Physics and Chemistry of Interfaces (John Wiley \& Sons, 2006).

${ }^{3}$ D. Quéré, Annu. Rev. Mater. Res. 38, 71 (2008).

${ }^{4}$ L. Bocquet and E. Lauga, Nat. Mater. 10, 334 (2011).

${ }^{5}$ X. Deng, L. Mammen, H.-J. Butt, and D. Vollmer, Science 335, 67 (2012).

${ }^{6}$ D. Quéré, Rep. Prog. Phys. 68, 2495 (2005).

${ }^{7}$ P. Papadopoulos, L. Mammen, X. Deng, D. Vollmer, and H.-J. Butt, Proc. Natl. Acad. Sci. U. S. A. 110, 3254 (2013).

${ }^{8}$ D. Richard, C. Clanet, and D. Quéré, Nature 417, 811 (2002).

${ }^{9}$ C. Antonini, A. Amirfazli, and M. Marengo, Phys. Fluids 24, 102104 (2012).

${ }^{10}$ S. Dash, M. T. Alt, and S. V. Garimella, Langmuir 28, 9606 (2012).

${ }^{11}$ J. C. Bird, R. Dhiman, H.-M. Kwon, and K. K. Varanasi, Nature 503, 385 (2013).

${ }^{12}$ C. Antonini, I. Bernagozzi, S. Jung, D. Poulikakos, and M. Marengo, Phys. Rev. Lett. 111, 014501 (2013).

${ }^{13}$ J. de Ruiter, R. Lagraauw, D. van den Ende, and F. Mugele, Nat. Phys. 11, 48 (2015).

${ }^{14}$ A. J. Meuler, J. D. Smith, K. K. Varanasi, J. M. Mabry, G. H. McKinley, and R. E. Cohen, ACS Appl. Mater. Interfaces 2, 3100 (2010).

${ }^{15}$ H. Lee, M. L. Alcaraz, M. F. Rubner, and R. E. Cohen, ACS Nano 7, 2172 (2013).

${ }^{16}$ J. B. Boreyko and C. P. Collier, ACS Nano 7, 1618 (2013).

${ }^{17}$ P. Eberle, M. K. Tiwari, T. Maitra, and D. Poulikakos, Nanoscale 6, 4874 (2014).

${ }^{18}$ T. Maitra, M. K. Tiwari, C. Antonini, P. Schoch, S. Jung, P. Eberle, and D. Poulikakos, Nano Lett. 14, 172 (2014).

${ }^{19}$ J. Lv, Y. Song, L. Jiang, and J. Wang, ACS Nano 8, 3152 (2014).

${ }^{20}$ N. A. Patankar, Soft Matter 6, 1613 (2010).

${ }^{21}$ C. Dietz, K. Rykaczewski, A. G. Fedorov, and Y. Joshi, Appl. Phys. Lett. 97, 033104 (2010).

${ }^{22}$ D. M. Anderson, M. K. Gupta, A. A. Voevodin, C. N. Hunter, S. A. Putnam, V. V. Tsukruk, and A. G. Fedorov, ACS Nano 6, 3262 (2012).

${ }^{23}$ A. Ghosh, S. Beaini, B. J. Zhang, R. Ganguly, and C. M. Megaridis, Langmuir 30, 13103 (2014). 
${ }^{24}$ A. T. Paxson, J. L. Yagüe, K. K. Gleason, and K. K. Varanasi, Adv. Mater. 26, 418 (2014).

${ }^{25}$ V. Bahadur and S. V. Garimella, Langmuir 23, 4918 (2007).

${ }^{26}$ D. 'tMannetje, S. Ghosh, R. Lagraauw, S. Otten, A. Pit, C. Berendsen, J. Zeegers, D. van den Ende, and F. Mugele, Nat. Commun. 5, 3559 (2014).

${ }^{27}$ P. Joseph, C. Cottin-Bizonne, J.-M. Benoît, C. Ybert, C. Journet, P. Tabeling, and L. Bocquet, Phys. Rev. Lett. 97, 156104 (2006).

${ }^{28}$ G. Mchale, N. J. Shirtcliffe, C. R. Evans, and M. I. Newton, Appl. Phys. Lett. 94, 064104 (2009).

${ }^{29}$ B. Bhushan, Beilstein J. Nanotechnol. 2, 66 (2011).

${ }^{30}$ I. U. Vakarelski, N. A. Patankar, J. O. Marston, D. Y. C. Chan, and S. T. Thoroddsen, Nature 489, 274 (2012).

${ }^{31}$ S. Srinivasan, W. Choi, K.-C. Park, S. S. Chhatre, R. E. Cohen, and G. H. McKinley, Soft Matter 9, 5691 (2013).

${ }^{32}$ B. R. Solomon, K. S. Khalil, and K. K. Varanasi, Langmuir 30, 10970 (2014).

${ }^{33}$ W. Xu, R. Leeladhar, Y. T. Kang, and C.-H. Choi, Langmuir 29, 6032 (2013).

${ }^{34}$ M. Dicuangco, S. Dash, J. A. Weibel, and S. V. Garimella, Appl. Phys. Lett. 104, 201604 (2014).

${ }^{35}$ M. Sheffer, A. Groysman, and D. Mandler, Corros. Sci. 45, 2893 (2003).

${ }^{36}$ W. Xu, H. Liu, S. Lu, J. Xi, and Y. Wang, Langmuir 24, 10895 (2008).

${ }^{37}$ I. Milošev, T. Kosec, and M. Bele, J. Appl. Electrochem. 40, 1317 (2010).

${ }^{38}$ T. Ishizaki, Y. Masuda, and M. Sakamoto, Langmuir 27, 4780 (2011).

${ }^{39}$ V. Kondrashov and J. Rühe, Langmuir 30, 4342 (2014).

${ }^{40}$ X. Feng and L. Jiang, Adv. Mater. 18, 3063 (2006).

${ }^{41}$ K. Liu and L. Jiang, Nanoscale 3, 825 (2011).

${ }^{42}$ G. Azimi, R. Dhiman, H.-M. Kwon, A. T. Paxson, and K. K. Varanasi, Nat. Mater. 12, 315 (2013).

${ }^{43}$ Y. Tian and L. Jiang, Nat. Mater. 12, 291 (2013).
${ }^{44}$ L. Martínez, E. Román, J. L. de Segovia, S. Poupard, J. Creus, and F. Pedraza, Appl. Surf. Sci. 257, 6202 (2011).

${ }^{45}$ N. J. Lawrence, K. Jiang, and C. L. Cheung, Chem. Commun. 47, 2703 (2011).

${ }^{46}$ G. Azimi, H.-M. Kwon, and K. K. Varanasi, MRS Commun. 4, 95 (2014).

${ }^{47}$ S. Zenkin, Š. Kos, and J. Musil, J. Am. Ceram. Soc. 97, 2713 (2014).

${ }^{48}$ S. Granick and S. C. Bae, Science 322, 1477 (2008).

${ }^{49}$ N. Giovambattista, P. G. Debenedetti, and P. J. Rossky, J. Phys. Chem. B 111, 9581 (2007).

${ }^{50}$ D. J. Preston, N. Miljkovic, J. Sack, R. Enright, J. Queeney, and E. N. Wang, Appl. Phys. Lett. 105, 011601 (2014).

${ }^{51}$ C. C. Chin, R. J. Lin, Y. C. Yu, C. W. Wang, E. K. Lin, W. C. Tsai, and T. Y. Tseng, Physica C 260, 86 (1996).

${ }^{52}$ P. Burroughs, A. Hamnett, A. F. Orchard, and G. Thornton, J. Chem. Soc., Dalton Trans. 17, 1686 (1976).

${ }^{53}$ E. Paparazzo, Mater. Res. Bull. 46, 323 (2011).

${ }^{54}$ M. Romeo, K. Bak, J. El Fallah, F. Le Normand, and L. Hilaire, Surf. Interface Anal. 20, 508 (1993).

${ }^{55}$ F. Zhang, P. Wang, J. Koberstein, S. Khalid, and S.-W. Chan, Surf. Sci. 563, 74 (2004).

${ }^{56}$ E. Bêche, P. Charvin, D. Perarnau, S. Abanades, and G. Flamant, Surf. Interface Anal. 40, 264 (2008).

${ }^{57}$ E. J. Preisler, O. J. Marsh, R. A. Beach, and T. C. McGill, J. Vac. Sci. Technol., B 19, 1611 (2001).

${ }^{58}$ V. F. Solovyov, T. Ozaki, A. Atrei, L. Wu, A. Al-Mahboob, J. T. Sadowski, X. Tong, D. Nykypanchuk, and Q. Li, Sci. Rep. 4, 4627 (2014).

${ }^{59}$ B. M. Reddy, P. Saikia, P. Bharali, Y. Yamada, T. Kobayashi, M. Muhler, and W. Grünert, J. Phys. Chem. C 112, 16393 (2008).

${ }^{60}$ J. Fan, X. Wu, L. Yang, and D. Weng, Catal. Today 126, 303 (2007). 